\title{
The fundamental relation between supermassive black holes and their host galaxies
}

\author{
Stefano de Nicola, ${ }^{1,2 \star}$ Alessandro Marconi ${ }^{3,4 \star}$ and Giuseppe Longo ${ }^{1,5,6}$ \\ ${ }^{1}$ Department of Physics 'E. Pancini', Università degli Studi di Napoli 'Federico II', Via Cinthia 6, I-80126 Napoli, Italy \\ ${ }^{2}$ Max-Planck-Institut für extraterrestrische Physik, Giessenbachstrasse 1, D-85748 Garching, Germany \\ ${ }^{3}$ Department of Physics and Astronomy, Università degli Studi di Firenze, Via G. Sansone 1, I-50019 Sesto Fiorentino, Firenze, Italy \\ ${ }^{4}$ INAF - Osservatorio Astrofisico di Arcetri, Largo Enrico Fermi 5, I-50125 Firenze, Italy \\ ${ }^{5}$ INFN, Napoli Unit, via Cinthia 6, I-80126 Napoli, Italy \\ ${ }^{6}$ Merle Kingsley Distinguished Visitor, CALTECH, Pasadena CA 90125, USA
}

Accepted 2019 August 21. Received 2019 August 15; in original form 2019 February 12

\begin{abstract}
We study the correlations between supermassive black holes (BHs) and their host galaxies, using a sample of $83 \mathrm{BH}$ masses collected from the most recent and reliable spatially resolved estimates available from the literature. We confirm the mono- and bivariate correlations between SMBHs and the bulges of their host galaxies, confirming that the correlation with the effective velocity dispersion is not significantly improved by higher dimensionality. Instead, pseudo-bulges do not seem to correlate with their SMBHs, probably because their secular evolution is often unable to trigger accretion onto the central BH. We then present a novel approach aimed at finding the fundamental relation between SMBHs and their host galaxies. For the first time, we analytically combine BH masses with the Fundamental Plane (FP), showing that $M_{\mathrm{BH}}-\sigma_{e}$ appears to be the fundamental relation rather than a putative 'BH Fundamental Plane' of the kind $M_{\mathrm{BH}}-\sigma_{e}-R_{e}$. These results can be explained by a picture which sees the $M_{\mathrm{BH}}-\sigma_{e}$ relation as a natural outcome of the change in AGN feedback from momentum-driven to energy-driven. The other scaling relations are then established through the FP.
\end{abstract}

Key words: Galaxy: fundamental parameters.

\section{INTRODUCTION}

The studies conducted in the last 25 yr have shown that supermassive black holes (hereafter SMBHs) play a crucial role in the formation and the evolution of their host spheroids (see Kormendy \& Ho 2013 and Graham 2016 for reviews). The most significant pieces of evidence are given by the correlations between the $\mathrm{BH}$ mass $M_{\mathrm{BH}}$ and the effective velocity dispersion $\sigma_{e}$ (Ferrarese \& Merritt 2000; Gebhardt et al. 2000; Tremaine et al. 2002; Gültekin et al. 2009) and that between $M_{\mathrm{BH}}$ and the bulge mass $M_{\text {bul }}$ (Magorrian et al. 1998; Marconi \& Hunt 2003; Häring \& Rix 2004) where, in the case of elliptical galaxies, the bulge corresponds to the whole spheroid. Other monovariate correlations which have been investigated are those with the bulge kinetic energy $M_{\mathrm{bul}} \sigma_{e}^{2}$ (Feoli \& Mancini 2009; Mancini \& Feoli 2012), the Dark Matter (DM) halo (Ferrarese 2002, but see Kormendy \& Bender 2011), the Sérsic index (Savorgnan et al. 2013), the pitch angle (Davis, Graham \& Seigar 2017) or that with the core radius (Saglia et al. 2016).

*E-mail: st.denicola2@gmail.com (SdN); alessandro.marconi@unifi.it (AM)
Recently, there have been studies investigating whether higher dimensionality, i.e. a relation combining $M_{\mathrm{BH}}$ with multiple galaxy parameters, can yield better $M_{\mathrm{BH}}$ predictions (e.g. Hopkins et al. 2007a,b; Sani et al. 2011; Beifiori et al. 2012; Saglia et al. 2016). Finding the fundamental relation between SMBHs and their hosts is of great importance since it would shed light on the physical mechanism behind these correlations and would provide us with the parameter(s) that yield(s) the most accurate $M_{\mathrm{BH}}$ predictions. Works focusing on $\mathrm{BH}$-galaxy scaling relations have shown that the $M_{\mathrm{BH}}-\sigma_{e}$ relation has the lowest intrinsic scatter (e.g. Gültekin et al. 2009; Saglia et al. 2016; van den Bosch 2016) and is just marginally (if at all) improved by higher dimensionality (Beifiori et al. 2012; Saglia et al. 2016). In a recent review, King \& Pounds (2015) show how this relation can be explained by a change in AGN feedback from momentum-driven to energy-driven and how it could generate the canonical $M_{\mathrm{BH}} \propto M_{\mathrm{bul}}^{1}$. Instead, in Hopkins et al. (2007b) it is found that the fundamental relation should be a plane of the kind $M_{\mathrm{BH}} \propto \sigma_{e}^{\alpha} R_{e}^{\beta}$ at $3 \sigma$ significance level. A total of five BH-galaxy bivariate correlations are detected in the very exhaustive study of Saglia et al. (2016), although Shankar et al. (2016), Shankar, Bernardi \& Sheth (2017), and Shankar et al. (2019) have raised the issue of a presence of a bias in favour of more massive BHs in their sample. 
The most general description of a bulge is given by the Fundamental Plane (hereafter FP, Djorgovski \& Davis 1987). This plane is given by the combination of the virial theorem and a tilt mostly given by the weak dependence of the mass-to-light ratio $M / L$ on $L$ itself (Cappellari et al. 2006). ${ }^{1}$ Thus, in order to have the most general picture of this $\mathrm{BH}$-bulge interaction, the whole FP should be combined with $\mathrm{BH}$ masses, also because the $\mathrm{BH}$ itself is part of the system probed by the FP. So far, there has been only one study (van den Bosch 2016) focusing on unifying the FP with BH masses. In that work, the author shows that a relation of the form $M_{\mathrm{BH}} \propto\left(\frac{L_{K}}{R_{e}}\right)^{3.8}$ should be used to measure $\mathrm{BH}$ masses when $\sigma_{e}$ measurements are not available. ${ }^{2}$

Besides classical bulges, we also encounter the so-called pseudobulges (Kormendy \& Kennicutt 2004; Fisher \& Drory 2008; Fisher et al. 2013). These systems actively form stars and are rotationally supported, thus resembling discs more than classical bulges. Our own Galaxy is the closest example of pseudo-bulge (Kormendy \& Ho 2013). Such systems do not seem to follow the BH-hosts scaling relations (Graham \& Scott 2013; Saglia et al. 2016) and may not even lie on the FP (Kormendy \& Kennicutt 2004, but see table 10 of Saglia et al. 2016), although these are quite difficult to identify (Graham 2014).

In this work, we analyse the existing correlations and we propose a novel multivariate analytic approach aimed at combining $\mathrm{BH}$ masses with the FP also taking into account covariances and correlations between observables. This enables us to verify, among all scaling relations and regardless of the dimensionality, which one yields the best predictions of the others. The paper is structured as follows. In Section 2, we present the data set. In Section 3, we fit linear regressions to our data. In Section 4, we present our approach aimed at unifying the FP and $\mathrm{BH}$ masses. In Section 5, we briefly examine the causality of our results and draw our conclusions. Notes on galaxies omitted from the analysis can be found in Appendix A. Unless differently specified, we will always provide $1 \sigma$ uncertainties on all variables.

\section{THE DATA}

\subsection{BH masses}

We start with the compilation of $97 \mathrm{BH}$ masses from Saglia et al. (2016). Then, we add three galaxies (NGC1277, IC2560, Cygnus A) from the Kormendy \& Ho (2013) compilation and other five galaxies (NGC1271, NGC1275, NGC1600, NGC3706, NGC5252) from van den Bosch (2016). Finally, we add the recent four BH mass estimates from Krajnović et al. (2018). All BH masses are measured through either stellar dynamics, gasdynamics, or astrophysical masers (Kormendy \& Ho 2013), thus deriving from spatially resolved kinematics. We do not consider BHs with upper-limits on their masses or estimates from reverberation mapping or virial methods, since these methods need to be calibrated with the $M_{\mathrm{BH}}-$ galaxy relations. This explains why our sample is a factor of 2 smaller than the largest BH masses compilations (Beifiori et al. 2012; van den Bosch 2016). We also discard BH masses from papers where observational details are not provided (e.g. Cappellari et al. 2008).

Two strong matters of debate are given by the inclusion of DM haloes (for stellar dynamics) and emission-line widths (for gas-

\footnotetext{
${ }^{1}$ The SMBH itself could contribute to this tilt, as we show in Section 4.

${ }^{2}$ Note that in this work the author focuses on whole galaxies rather than on bulges solely.
}

dynamics) in the analysis when estimating $M_{\mathrm{BH}}$. In both cases, the authors claim that neglecting these factors can yield underestimated $M_{\mathrm{BH}}$ values, even if in the first case not including the DM halo in the analysis only indirectly affects $M_{\mathrm{BH}}$ through $M / L$ (Kormendy \& Ho 2013). A DM halo is not always important [see e.g. table 1 of Schulze \& Gebhardt (2011) or table 3 of Rusli et al. (2013)], especially if the $\mathrm{BH}$ sphere of influence is well resolved, while in the second case it is not guaranteed that the emission-line widths contribute significantly to the analysis, as they could simply be due to unresolved rotation (which is taken into account in the modelling) or turbulent motions. In this sample, we find several galaxies having their $M_{\mathrm{BH}}$ estimated both with and without modelling a DM halo. When possible, we try to be conservative, keeping those estimates which take a DM halo into account (for stellar dynamics), or emission-line widths (for gasdynamics) in the analysis. Details on our omissions are discussed in Appendix A. This leaves us with a total of 83 galaxies (see Table 1).

\subsection{Galaxy parameters}

Effective velocity dispersions are obtained from the same literature sources providing $M_{\mathrm{BH}}$ (Kormendy \& Ho 2013; Saglia et al. 2016; van den Bosch 2016). These are measured according to the equation

$\sigma=\left(\frac{\int_{0}^{R_{e}} \sqrt{\left(v(r)^{2}+\sigma(r)^{2}\right)} I(r) d r}{\int_{0}^{R_{e}} I(r) d r}\right)^{\frac{1}{2}}$

where $v(r)$ and $\sigma(r)$ are the first two moments of the collisionless Boltzmann equation, $I(r)$ is the surface brightness profile, and $R_{e}$ is the effective radius. We are convinced that the $\mathrm{BH}$ sphere of influence (hereafter SOI) should be included in the computation of $\sigma_{e}$ since the BH itself is part of the system probed by the FP. Other authors (e.g. McConnell \& Ma 2013) prefer to omit this region, in order to (try to) decouple the gravitational effects of the SMBH from the $\sigma_{e}$ estimate.

We take the $3.6 \mu \mathrm{m}$ Spitzer photometry from Savorgnan \& Graham (2016, for the effective radii) and Savorgnan et al. (2016, for the luminosities). In the first paper, the authors perform sophisticated decompositions, claiming not to underestimate the systematics involved in such analysis. We convert effective radii to physical units using distances of our sample. In order to alleviate the problem given by incomplete data, when Spitzer photometry is not available we turn to the $K$-band photometry from 2MASS data performed by van den Bosch (2016). However, in this last work the focus is not on decompositions and hence such data can only be used for early types. Moreover, these data are less deep than Spitzer's and the photometric analysis is performed in a much more simplified manner with respect to the work of Savorgnan \& Graham (2016). Nevertheless, in Fig. 1 we compare Spitzer and 2MASS data. For the luminosities, the agreement is fairly good $(\sim 0.12 \mathrm{dex})$, as can be expected given the low $K-3.6$ colour index (Sani et al. 2011), while things are slightly worse when dealing with radii $(\sim 0.20 \mathrm{dex})$, which can be explained by the different analyses and techniques used to derive the photometric variables (Savorgnan \& Graham 2016; van den Bosch 2016).

\section{LINEAR REGRESSIONS}

The first step of the analysis consists in fitting the scaling relations to our data. These relations have the form

$z=\alpha(x-\langle x\rangle)+\gamma+\varepsilon$ 
Table 1. Column 1: Galaxy name. Column 2: Morphology. Column 3: Flag $A, A=0$ indicates core ellipticals, $A=1$ indicates power-law ellipticals, $A=2$ indicates bulges, $A=3$ indicates pseudo-bulges (Saglia et al. 2016). Column 4: Distance. Column 5: BH mass. Column 6: Effective velocity dispersion. Column 7: Luminosity, measured either at $3.6 \mu \mathrm{m}$ (Savorgnan et al. 2016) or from 2MASS data (van den Bosch 2016, Section 2.2. Column 8: Effective radius, coming from the same analysis as L. Column 9: Flag $B, B=0$ indicates $K$-band data, $B=1$ indicates Spitzer data.

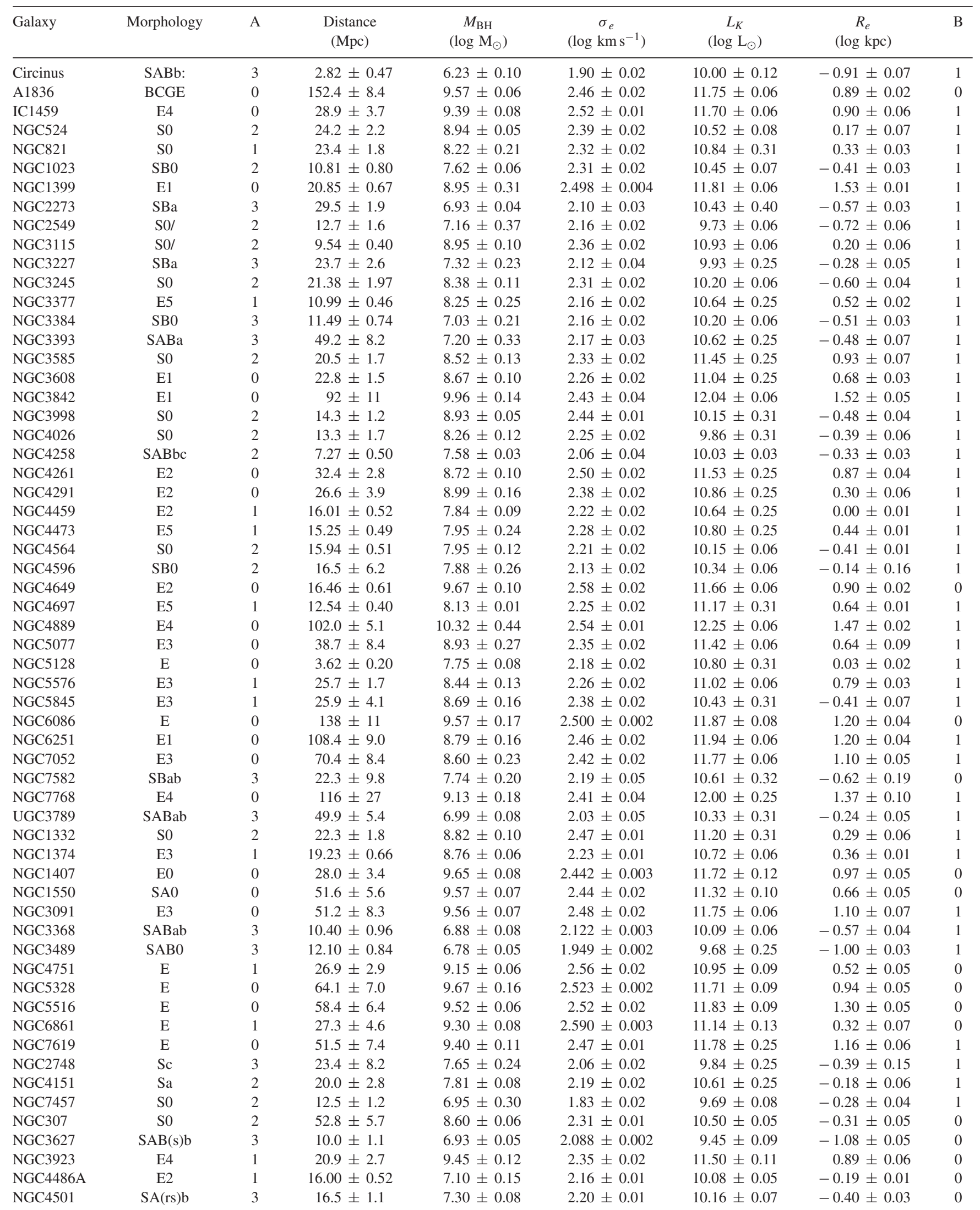


Table 1 - continued

\begin{tabular}{|c|c|c|c|c|c|c|c|c|}
\hline Galaxy & Morphology & A & $\begin{array}{l}\text { Distance } \\
(\mathrm{Mpc})\end{array}$ & $\begin{array}{c}M_{\mathrm{BH}} \\
\left(\log \mathrm{M}_{\odot}\right)\end{array}$ & $\begin{array}{c}\sigma_{e} \\
\left(\log \mathrm{km} \mathrm{s}^{-1}\right)\end{array}$ & $\begin{array}{c}L_{K} \\
\left(\log L_{\odot}\right)\end{array}$ & $\begin{array}{c}R_{e} \\
(\log \mathrm{kpc})\end{array}$ & B \\
\hline NGC5018 & E3 & 1 & $40.6 \pm 4.9$ & $8.02 \pm 0.08$ & $2.32 \pm 0.01$ & $11.54 \pm 0.09$ & $0.62 \pm 0.05$ & 0 \\
\hline IC4296 & BCGE & 0 & $49.2 \pm 3.6$ & $9.11 \pm 0.07$ & $2.51 \pm 0.02$ & $11.78 \pm 0.25$ & $1.21 \pm 0.03$ & 1 \\
\hline NGC1277 & $\mathrm{S} 0 /$ & 2 & $73.0 \pm 7.3$ & $9.70 \pm 0.05$ & $2.52 \pm 0.07$ & $10.83 \pm 0.08$ & $0.09 \pm 0.04$ & 0 \\
\hline IC2560 & $\mathrm{SBbc}$ & 3 & $33.2 \pm 3.3$ & $6.59 \pm 0.16$ & $2.15 \pm 0.02$ & $10.13 \pm 0.25$ & $-0.14 \pm 0.04$ & 1 \\
\hline NGC3031 & $\mathrm{Sb}$ & 2 & $3.60 \pm 0.13$ & $7.81 \pm 0.13$ & $2.15 \pm 0.02$ & $10.43 \pm 0.31$ & $-0.24 \pm 0.02$ & 1 \\
\hline NGC4374 & E1 & 0 & $18.51 \pm 0.60$ & $8.97 \pm 0.05$ & $2.47 \pm 0.02$ & $11.64 \pm 0.25$ & $1.07 \pm 0.01$ & 1 \\
\hline NGC4486 & E1 & 0 & $16.68 \pm 0.62$ & $9.68 \pm 0.04$ & $2.51 \pm 0.03$ & $11.64 \pm 0.25$ & $0.85 \pm 0.02$ & 1 \\
\hline NGC4594 & $\mathrm{Sa}$ & 2 & $9.87 \pm 0.82$ & $8.82 \pm 0.04$ & $2.38 \pm 0.02$ & $10.79 \pm 0.25$ & $-0.03 \pm 0.08$ & 1 \\
\hline NGC3379 & E1 & 0 & $10.70 \pm 0.54$ & $8.62 \pm 0.11$ & $2.31 \pm 0.02$ & $10.96 \pm 0.25$ & $0.42 \pm 0.02$ & 1 \\
\hline NGC221 & E2 & 1 & $0.80 \pm 0.03$ & $6.39 \pm 0.19$ & $1.89 \pm 0.02$ & $9.12 \pm 0.04$ & $-0.90 \pm 0.02$ & 0 \\
\hline NGC5252 & So & 2 & $92.0 \pm 9.2$ & $8.98 \pm 0.23$ & $2.28 \pm 0.02$ & $11.49 \pm 0.09$ & $0.88 \pm 0.06$ & 0 \\
\hline NGC4339 & $\mathrm{E}$ & 1 & $16.0 \pm 1.6$ & $7.63 \pm 0.36$ & $1.98 \pm 0.02$ & $10.26 \pm 0.25$ & $0.37 \pm 0.04$ & 0 \\
\hline NGC4434 & $\mathrm{E}$ & 1 & $22.4 \pm 2.2$ & $7.85 \pm 0.15$ & $1.99 \pm 0.02$ & $10.28 \pm 0.25$ & $0.20 \pm 0.04$ & 0 \\
\hline NGC4578 & $\mathrm{E}$ & 1 & $16.3 \pm 1.6$ & $7.28 \pm 0.22$ & $2.03 \pm 0.02$ & $10.33 \pm 0.25$ & $0.49 \pm 0.04$ & 0 \\
\hline NGC4762 & $\mathrm{E}$ & 1 & $22.6 \pm 2.3$ & $7.36 \pm 0.14$ & $2.13 \pm 0.02$ & $11.05 \pm 0.25$ & $1.06 \pm 0.04$ & 0 \\
\hline
\end{tabular}
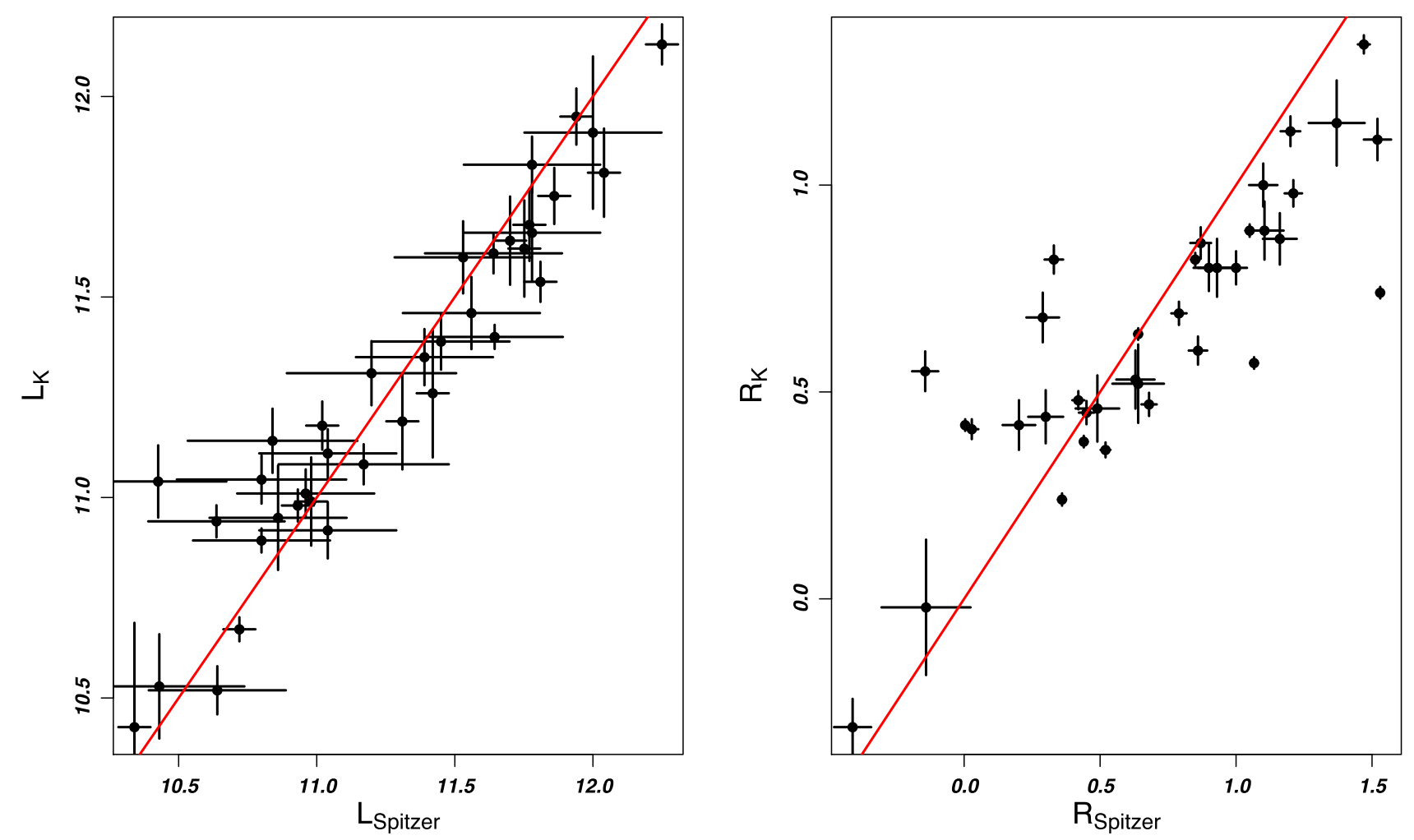

Figure 1. Comparison of the $K$-band and the Spitzer photometries for the galaxies of our sample for which both measurements are available. The red lines are the 1:1 lines. The values are these revised plots to the productuin good agreement with the Spitzer radii being on average slightly larger, which can be expected since Spitzer data are deeper. 
for monovariate correlations and

$z=\alpha(x-\langle x\rangle)+\beta(y-\langle y\rangle)+\gamma+\varepsilon$

for bivariate correlations. In these equations, $\alpha$ and $\beta$ are the slopes, $\gamma$ is the zero-point, and $\varepsilon$ is the intrinsic scatter around the dependent variable. This last parameter is of great importance since it represents the scatter not due to measurement errors, thus providing information about which variable(s) is (are) most closely connected to the central $\mathrm{BH}$. Centring the independent variable reduces the covariance between the observables; moreover, the zero-point should be $\sim\langle z\rangle$. Commonly used fitting routines are the Bayesian linmixerr and its multidimensional equivalent $\mathrm{ml}$ inmixerr (Kelly 2007). Here, we rely on the robust lts_linefit and lts_planefit algorithms (Cappellari et al. 2013), which combine the Least Trimmed Squares algorithm from Rousseeuw \& Van Driessen (2006) with a residuals sum-of-squares minimization. These routines can automatically exclude the outliers from the fit, but we decided not to use this feature. For example, the largest galaxies (e.g. NGC4889, NGC1600, see Fig. 2) are expected to be outliers of the $M_{\mathrm{BH}}-\sigma_{e}$ relation because of the longer time needed by their SMBH to clear the bulge of gas, which results in abnormally large $M_{\mathrm{BH}}$ 's (King \& Pounds 2015). All the variables are logarithmic with units of measurement reported in Table 1. In all fits, the dependent variable will be $M_{\mathrm{BH}}$.

We consider the monovariate correlations between $M_{\mathrm{BH}}$ and $\sigma_{e}, L_{K}$, and $R_{e}$. Then, we turn to bivariate correlations between $M_{\mathrm{BH}}$ and three possible pairs of galaxy parameters. The relation between $M_{B H}, \sigma_{e}$, and $R_{e}$ is of particular interest since it was proposed as the fundamental relation by Hopkins et al. (2007b). In the exhaustive study of Saglia et al. (2016), this correlation is also detected, but with a stronger dependence on $\sigma_{e}$ and a slightly weaker dependence on $R_{e}$.

\subsection{Regression results}

Regression results are reported in Table 2, and shown in Figs 2 (monovariate) and 3 (bivariate). Those points interpreted as unreliable data (see Appendix A) are plotted as red points and omitted from the regressions. Galaxies are divided into four subgroups (core ellipticals, power-law ellipticals, spirals with classical bulges and pseudo-bulges) using the $T$ flag defined in table 1 of Saglia et al. (2016). We notice that $M_{\mathrm{BH}}-\sigma_{e}$ has the lowest intrinsic scatter, whose value agrees with those found in similar studies (Saglia et al. 2016; Savorgnan et al. 2016). Using a sample of 45 early types, Kormendy \& Ho (2013) derive a relation with a scatter $<0.3$ dex. Instead, BHs correlate much more weakly with the bulge photometric parameters and, interestingly, the relations with luminosity and effective radius show the same slope. Since the intrinsic scatter embeds all factors not accountable with measurement errors, it appears that SMBHs are indeed more closely connected to $\sigma_{e}$ than any other variable, confirming the conclusions of earlier works on this topic (Gültekin et al. 2009; Beifiori et al. 2012; van den Bosch 2016).

At variance with their classical counterparts, pseudo-bulges do not seem to correlate with their central BHs (Kormendy, Bender \& Cornell 2011), except for a possible correlation with $\sigma_{e}$. It is intriguing that in Saglia et al. (2016) this correlation is not detected (see their table 11), even if their sample constitutes the basis of our own compilation. ${ }^{3}$ It appears that the limited number of pseudo-

${ }^{3}$ Note that we discard several galaxies that are taken into account in the Saglia et al. (2016) analysis. See Appendix A. bulges with reliable $\mathrm{BH}$ masses detections prevents us from reaching a definitive conclusion. Moreover, both classical and pseudo-bulges are not uniquely defined and several galaxies might host both components (Erwin et al. 2015).

It should be stressed that the large range spanned by $\mathrm{BH}$ masses can yield misleading results. In fig. 11 of King \& Pounds (2015), it is shown how similar slopes but different normalizations for different galaxy subgroups can give anomalously high slopes. In Table 3, we fit the $M_{\mathrm{BH}}-\sigma_{e}$ relation to each of the four subgroups, showing that slopes are much closer to the value of 4 expected from momentumdriven theories, which do not constitute a serious threat for the bulge integrity. Since our BH mass estimates are biased towards higher values because of the limited resolution of current-days telescopes (Bernardi et al. 2007; Shankar et al. 2016), the slope of this relation could naturally increase with data from new generation telescopes, if the sample is very heterogeneous in masses.

As shown in Table 2, combining $\sigma_{e}$ with $L$ or $R_{e}$ does not significantly improve the intrinsic scatter of the $M_{\mathrm{BH}}-\sigma_{e}$ relation. Nevertheless, neither $L$ nor $R_{e}$ have a slope consistent with zero, even at $3 \sigma$ limit. Our results are consistent with the findings of Saglia et al. (2016). ${ }^{4}$ The dependence on $\sigma_{e}$ is stronger than what originally found by Hopkins et al. (2007b) and, interestingly, the $\sigma_{e}$ coefficient is consistent with the value of 4 predicted by momentumdriven AGN feedback (King 2003).

\section{THE BH HYPERPLANE: A MULTIVARIATE ANALYTIC APPROACH}

This section describes a novel analytic approach that combines $M_{\mathrm{BH}}$ with FP. The only other work published so far which deals with this issue is van den Bosch (2016), where the author shows that, when $\sigma_{e}$ measurements are not available, the ratio $L_{K} / R_{e}$ should be used as a proxy of $M_{\mathrm{BH}}$. Here, we want to verify which relation gives the best prediction of the others, i.e. able to reproduce their slopes and intrinsic scatters. Although we know that FP does not improve if additional parameters are added (Djorgovski \& Davis 1987), in order to find the fundamental $\mathrm{BH}$-hosts relation, this plane must be taken into account since it provides the most general description of a host bulge. Moreover, the BH itself could contribute to the tilt in the FP (see van den Bosch 2016). First, we model the FP with a trivariate Gaussian following Bernardi et al. (2003b). Then, we show how to use this description to find the fundamental relation. Since pseudo-bulges do not seem to follow the scaling relations, we have preferred to omit them from the sample. Results including these systems are reported in Appendix B, where we show that our conclusions do not differ significantly.

\subsection{A four-dimensional regression}

We start writing down the most general relation between $M_{\mathrm{BH}}$ and the host galaxy: ${ }^{5}$

$M_{\mathrm{BH}}=\alpha L+B R_{e}+C V+g_{0} \Sigma$

which is shown in Fig. 4 for our sample. All the variables of appearing in this equation $\left(M_{\mathrm{BH}}, L, V\right.$, and $\left.R_{e}\right)$ are logarithmic.

\footnotetext{
${ }^{4}$ That authors use masses rather than luminosities. These are computed from mass-to-light ratios taken from different sources. See appendix B of Saglia et al. (2016).

${ }^{5}$ To avoid confusion with the standard deviation of the Gaussians, we will use $V$ to label the velocity dispersion in the remainder of the paper.
} 

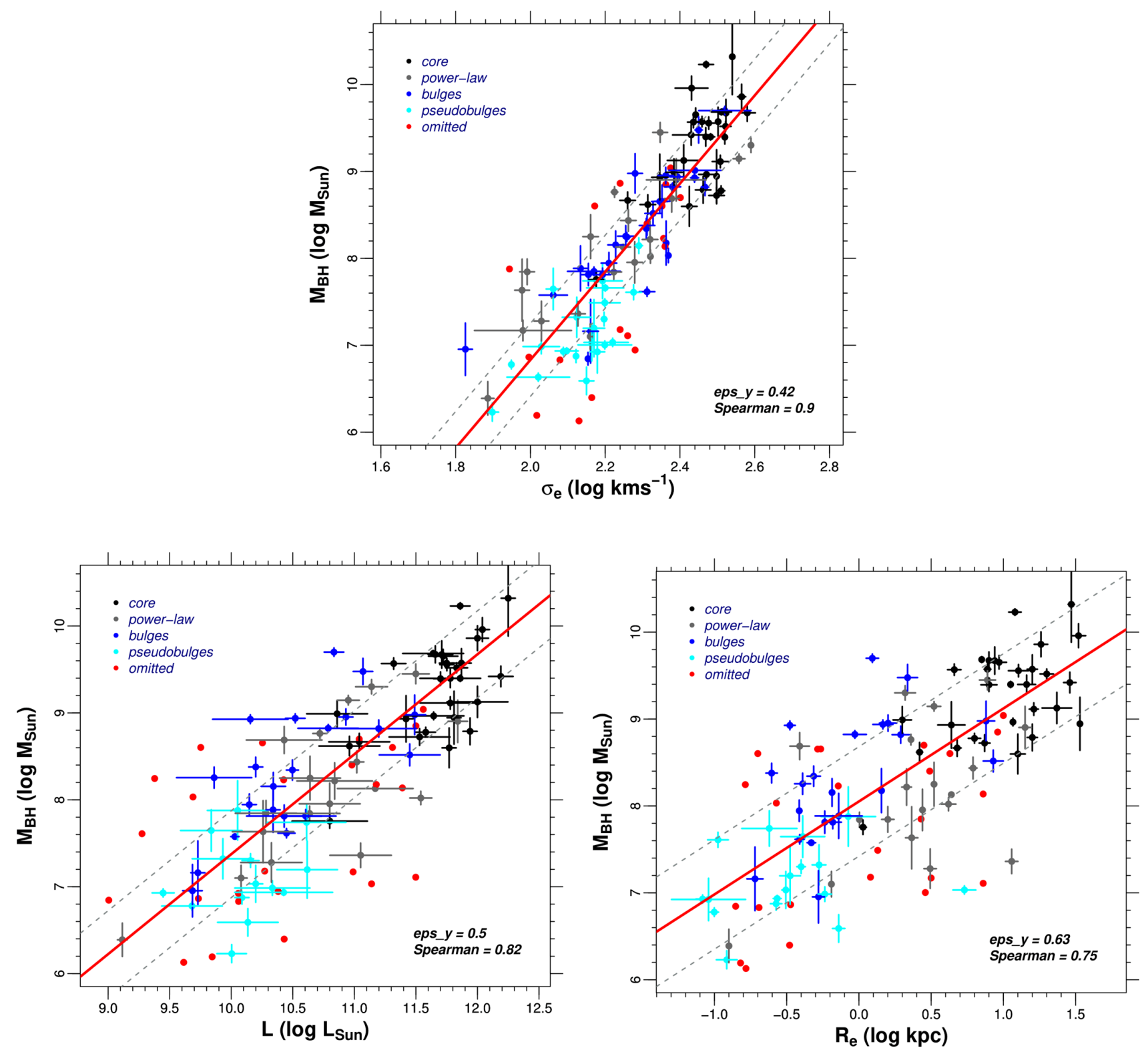

Figure 2 Monovariate correlations between BH masses and galaxy parameters (upper row: $\sigma_{e}$, lower row, left: L, lower row, right: $R_{e}$ ). Galaxies are coloured according to the $T$ flag defined in table 1 of Saglia et al. (2016, Column 2 of table 1). Red points are omitted from the regressions (see Appendix A). The intrinsic scatter and the Spearman's coefficient are printed on the bottom-right of the plot. The dashed lines delimit the range given by the intrinsic scatter.

Here, $\Sigma$ is the dispersion of this relation and $g_{0}$ a random Gaussian number with zero mean, so that the product of $\Sigma$ and $g_{0}$ represents the intrinsic scatter of our relation. ${ }^{6}$

The first thing to do is fit equation (4) to our data, in order to check if we are able to constrain the three slopes of the hyperplane and its intrinsic scatter. This is needed not only to see how the coefficients compare to those obtained for lower dimensionality relations but also to quantify the effect of the FP in establishing such correlations. The regression has been performed by extending the fitting routine used in the previous section (Cappellari et al. 2013) to the four-dimensional case. We obtain

$A=-0.12 \pm 0.33$

${ }^{6}$ Note that there should also be a dependence on redshift, but since for our sample $D_{\max } \sim 250 \mathrm{Mpc}$ (Cygnus A), this term is negligible.

$$
\begin{aligned}
& B=0.56 \pm 0.33 ; \\
& C=4.18 \pm 0.48 ; \\
& \Sigma=0.35 \pm 0.04 ; \\
& N=8.34 \pm 0.05,
\end{aligned}
$$

where $N$ is the zero-point of the regression. The errors are only slightly larger than those obtained with planar regressions, which is reassuring because the dimensionality of the problem combined with the low number of points could have yielded abnormally large errors, or even prevented the convergence of the algorithm.

We see that the intrinsic scatter of this relation is comparable with those of the other regressions where $\sigma_{e}$ is involved (see column 6 of Table 2), resulting slightly lower than the BHFPs with $\sigma_{e}$ 
Table 2. Regression results between $M_{\mathrm{BH}}$ and the galaxy parameters. Column 1: The independent variable(s). Column 2: The sample used in the regression, All for the full sample, ClBul of classical bulges and Pseudo for pseudo-bulges. Columns 3 and 4: The slopes $(\alpha$ and $\beta$ ). Column 5: The zero-point $(\gamma)$. Column 6: The intrinsic scatter $(\varepsilon)$. Columns 7 and 8: The values of $\langle x\rangle$ and $\langle y\rangle$ (see equations 2 and 3).

\begin{tabular}{lccccccc}
\hline Variable(s) & Subgroup & $\alpha$ & $\beta$ & $\gamma$ & $\varepsilon$ & $\langle x\rangle$ & $\langle y\rangle$ \\
\hline$\sigma_{e}$ & All & $5.07 \pm 0.27$ & - & $8.30 \pm 0.05$ & $0.42 \pm 0.04$ & 2.291 & - \\
& ClBul & $4.48 \pm 0.30$ & - & $8.60 \pm 0.05$ & $0.38 \pm 0.04$ & 2.333 & - \\
& Pseudo & $3.50 \pm 0.70$ & - & $7.14 \pm 0.07$ & $0.27 \pm 0.08$ & 2.135 & - \\
$L$ & All & $1.12 \pm 0.08$ & - & $8.48 \pm 0.06$ & $0.53 \pm 0.05$ & 10.913 & - \\
& ClBul & $1.00 \pm 0.09$ & - & $8.71 \pm 0.06$ & $0.47 \pm 0.05$ & 11.074 & - \\
& Pseudo & $0.49 \pm 0.47$ & - & $7.10 \pm 0.12$ & $0.43 \pm 0.14$ & 10.109 & - \\
$R_{e}$ & All & $1.07 \pm 0.10$ & - & $8.43 \pm 0.07$ & $0.63 \pm 0.06$ & 0.306 & - \\
& ClBul & $0.91 \pm 0.12$ & - & $8.70 \pm 0.07$ & $0.60 \pm 0.06$ & 0.500 & - \\
& Pseudo & $0.15 \pm 0.23$ & - & $7.09 \pm 0.10$ & $0.39 \pm 0.12$ & -0.502 & - \\
$\sigma_{e}-R_{e}$ & All & $3.95 \pm 0.34$ & $0.39 \pm 0.09$ & $8.43 \pm 0.05$ & $0.38 \pm 0.04$ & 2.301 & 0.310 \\
& ClBul & $3.69 \pm 0.34$ & $0.32 \pm 0.09$ & $8.68 \pm 0.05$ & $0.34 \pm 0.04$ & 2.341 & 0.500 \\
& Pseudo & $2.80 \pm 0.83$ & $-0.14 \pm 0.19$ & $7.05 \pm 0.08$ & $0.27 \pm 0.11$ & 2.120 & -0.529 \\
$\sigma_{e}-L$ & All & $3.48 \pm 0.43$ & $0.43 \pm 0.11$ & $8.48 \pm 0.05$ & $0.37 \pm 0.04$ & 2.303 & 10.923 \\
& ClBul & $3.20 \pm 0.45$ & $0.37 \pm 0.11$ & $8.69 \pm 0.05$ & $0.34 \pm 0.04$ & 2.341 & 11.074 \\
& Pseudo & $2.9 \pm 1.3$ & $-0.08 \pm 0.46$ & $7.01 \pm 0.10$ & $0.30 \pm 0.13$ & 2.096 & 10.113 \\
\hline
\end{tabular}
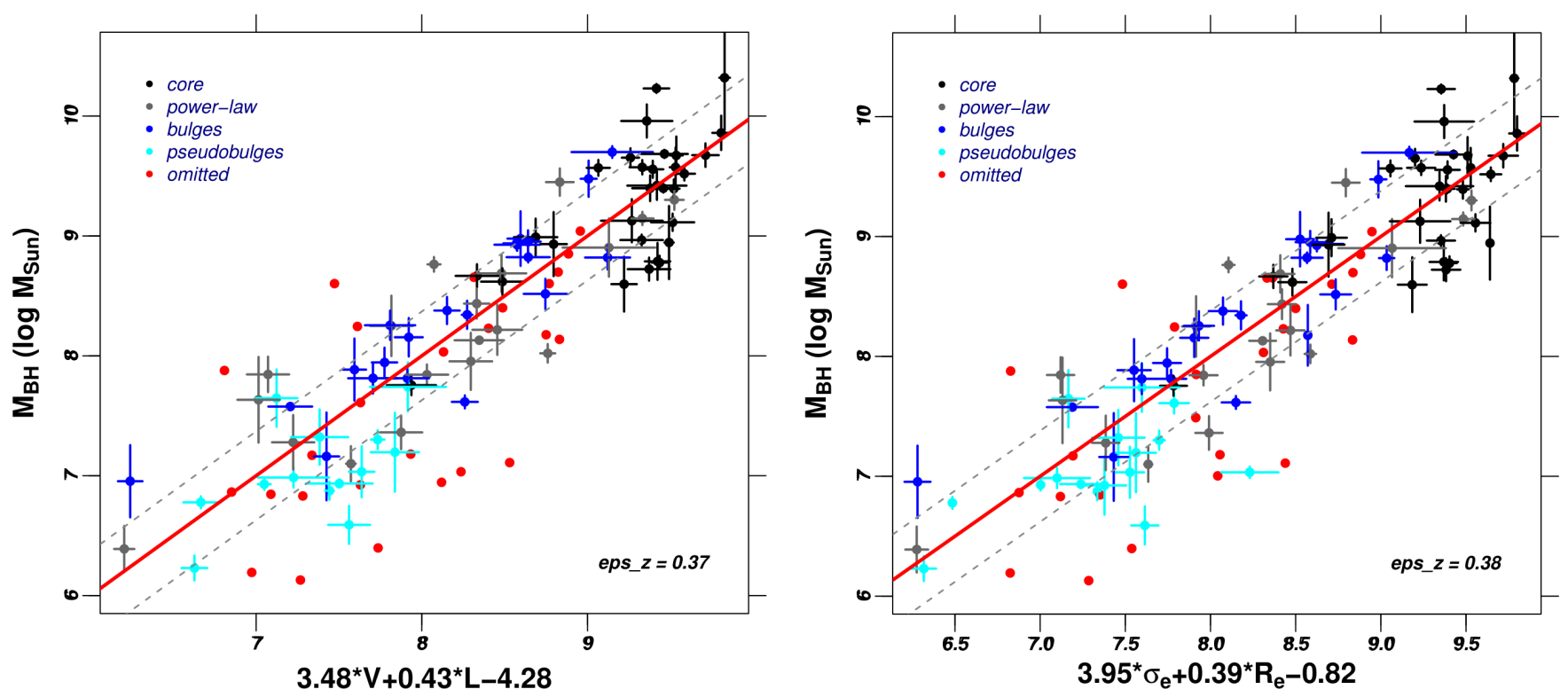

Figure 3. Bivariate correlations between $\mathrm{BH}$ masses and galaxy parameters (left: $M_{\mathrm{BH}}-\sigma_{e}-L$, right: $\left.M_{\mathrm{BH}}-\sigma_{e}-R_{e}\right)$. Symbols and colour coding are the same as in Fig. 2.

Table 3. Results of the fit of the $M_{\mathrm{BH}}-\sigma_{e}$ relation to each subgroup defined in Saglia et al. (2016, see also Section 3.1). Column 1: The subgroup. Column 2 and 3: The slopes and the zero-points. Column 4: The intrinsic scatter. Column 5: The values of $\langle x\rangle$ (see equation 2). All slopes are closer to the value of 4 predicted by momentum-driven theories (King \& Pounds 2015) than that obtained from the full sample. Note the much larger uncertainty on the Pseudo-slope.

\begin{tabular}{lcccc}
\hline Subgroup & $\alpha$ & $\gamma$ & $\varepsilon$ & $\langle x\rangle$ \\
\hline Core & $4.32 \pm 0.89$ & $9.27 \pm 0.07$ & $0.38 \pm 0.07$ & 2.404 \\
Power & $3.65 \pm 0.55$ & $8.09 \pm 0.10$ & $0.41 \pm 0.10$ & 2.270 \\
ClBul & $4.25 \pm 0.54$ & $8.28 \pm 0.07$ & $0.34 \pm 0.07$ & 2.283 \\
Pseudo & $3.50 \pm 0.70$ & $7.14 \pm 0.07$ & $0.27 \pm 0.08$ & 2.135 \\
\hline
\end{tabular}

and consistent with the value found for $M_{\mathrm{BH}}-\sigma_{e}$ within $1.5 \sigma$. The comparison between the scatters is made by evaluating the quantity

$$
\frac{\left|\varepsilon_{1}-\varepsilon_{2}\right|}{\sqrt{\sigma_{\varepsilon_{1}}^{2}+\sigma_{\varepsilon_{2}}^{2}}} \text {. }
$$

Once again, it appears that $\sigma_{e}$ alone is a very good predictor of $M_{\mathrm{BH}}$. Interestingly, the introduction of $L$ does not significantly alter the slopes of the $M_{\mathrm{B}}-\sigma_{e}-R_{e}$, indeed the $L$-slope is the only one consistent with zero within $1 \sigma$.

\subsection{Modelling the BH hyperplane}

We now investigate the effects of the FP on the relations between $\mathrm{BH}$ and bulge structural parameters. The three variables defining the FP 


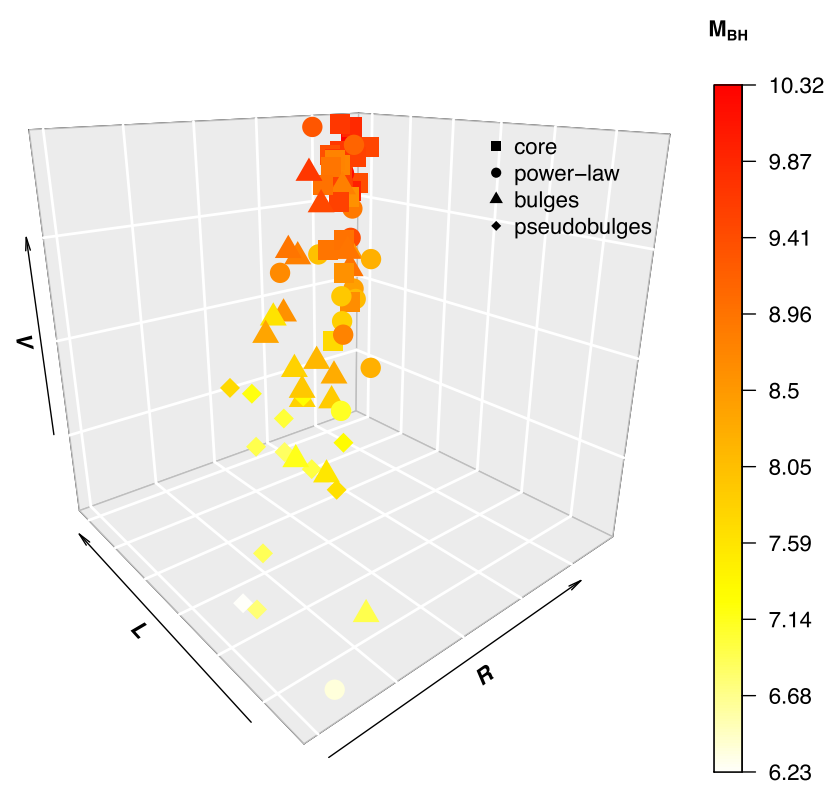

Figure 4. The 3D representation of the putative 'BH hyperplane'. Different symbols denote different galaxy subsets, as shown in the legend. Points are coloured according to their $M_{\mathrm{BH}}$ values.

are strongly pairwise correlated (Bernardi et al. 2003b). Due to the smallness of our sample, computing covariances and correlations between these three observables may be cumbersome. Thus, we turn to the much more robust analysis of Bernardi et al. (2003b).

In that work, the authors studied a sample of $~ 9000$ early-type galaxies from the SDSS finding that the luminosity distribution is well modelled by a Gaussian (see also Saglia et al. 2001), and also the distributions of both $V$ and $R_{e}$ around the mean (at fixed $L$ ) are of Gaussian shape. This means that

$\phi\left(L, R_{e}, V\right)=\psi\left(R_{e}, V \mid L\right) \phi(L)$,

where $\psi\left(R_{e}, V \mid L\right)$ and $\phi(L)$ are a bivariate and a monovariate Gaussian, respectively, and $\phi\left(L, R_{e}, V\right)$ is the joint distribution, which is well modelled by a trivariate Gaussian. In practice, we draw $L$ from a Gaussian distribution with mean $\langle L\rangle$ and variance $\sigma_{L}^{2}$ then $R_{e}$ from a Gaussian with mean $\left\langle R_{e} \mid L\right\rangle$ and variance $\sigma_{R_{e} \mid L}^{2}$ and finally the velocity dispersion taking into account both correlations with $L$ and $R_{e}$. Labelling the correlation coefficients between two variables with $\rho$, we obtain (see appendix A of Bernardi et al. 2003b)

$L=g_{1} \sigma_{L}$

$R_{e}=\frac{L}{\sigma_{L}} \sigma_{R_{e}} \rho_{R_{e} L}+g_{1} \sigma_{R_{e}} \sqrt{1-\rho_{R_{e} L}^{2}}$

$V=\frac{L}{\sigma_{L}} \xi_{L V}+\frac{R_{e}}{\sigma_{R_{e}}} \xi_{R_{e} V}+g_{2} \sigma_{V \mid R_{e} L}$,

where the $g$ s are Gaussian random numbers with zero mean and unit variance,

$\xi_{L V}=\sigma_{V} \frac{\rho_{L V}-\rho_{R_{e} L} \rho_{R_{e} V}}{1-\rho_{L R_{e}}^{2}}$
$\xi_{R_{e} V}=\sigma_{V} \frac{\rho_{R_{e} V}-\rho_{R_{e} L} \rho_{L V}}{1-\rho_{L R_{e}}^{2}}$
Table 4. The covariance matrix describing the FP of the sample of Bernardi et al. (2003b). This is derived from $z$-band observations.

\begin{tabular}{lc}
\hline Coefficient & Value \\
\hline$\sigma_{V}$ & 0.17 \\
$\sigma_{L}$ & 0.69 \\
$\sigma_{R_{e}}$ & 0.64 \\
$\rho_{V L}$ & 0.78 \\
$\rho_{V R_{e}}$ & 0.54 \\
$\rho_{R_{e} L}$ & 0.88 \\
\hline
\end{tabular}

$\sigma_{V \mid L R_{e}}=\sigma_{V} \sqrt{\frac{1-\rho_{L R_{e}}^{2}-\rho_{L V}^{2}-\rho_{V R_{e}}^{2}+2 \rho_{R_{e} L} \rho_{L V} \rho_{R_{e} V}}{1-\rho_{L R_{e}}^{2}}}$

and

$\mathscr{C}=\left(\begin{array}{ccc}\sigma_{L} & \rho_{R_{e} L} \sigma_{R_{e}} \sigma_{L} & \rho_{L V} \sigma_{L} \sigma_{V} \\ \rho_{R_{e} L} \sigma_{R_{e}} \sigma_{L} & \sigma_{R_{e}} & \rho_{R_{e} V} \sigma_{R_{e}} \sigma_{V} \\ \rho_{L V} \sigma_{L} \sigma_{V} & \rho_{R_{e} V} \sigma_{R_{e}} \sigma_{V} & \sigma_{V}\end{array}\right)$

is the covariance matrix. The parameters of this matrix should be estimated using a maximum likelihood analysis: since we expect our data to be distributed following a trivariate normal distribution

$\mathscr{L}=\mathscr{N}^{3 D}\left(\left\{\boldsymbol{L}, \boldsymbol{V}, \boldsymbol{R}_{\boldsymbol{e}}\right\}, \mathscr{C}\right)$,

where $\mathscr{C}$ is the covariance matrix, we can use this function to estimate the six parameters that define $\mathscr{C}$ needed to determine our best-fitting function. However, since our sample is not very large, we speculate that these six parameters might be so well constrained. In order to increase the robustness of the analysis, we can take the covariance matrix derived from the sample of Bernardi et al. (2003b). In fact, that covariance matrix describes the properties of a generic sample of early types. If we consider only the early types in our sample, then we should, in principle, deal with a (biased) subset of that sample. The problem is that our photometric data are either at $3.6 \mu \mathrm{m}$ or in the $K$-band, but such a covariance matrix is not available for that bands. To alleviate the problem given by the fact that the SDSS observations are carried out in the optical, we take the covariance matrix from Bernardi et al. (2003b) in the $z$ band (see Table 4), thus assuming that both variances and correlations do not change significantly.

As a test to ensure that our FP is consistent with that derived in Bernardi et al. (2003c), we plot in the left-hand panel of Fig. 5 the early types of our sample with their best-fitting line. Our galaxies seem to follow that FP but are, on average, larger than expected. This is a selection effect generated by the need of large galaxies to resolve the BH SOIs, otherwise the required resolution is beyond current-day facilities (Bernardi et al. 2007; Shankar et al. 2016). In order to remove the bias, we estimated the normalization through a one-dimensional regression fixing the slopes to the FP of Bernardi et al. (2003c) and then computed the residuals with respect to the new best-fitting line.

The residuals distribution is plotted in the right-hand panel of Fig. 5. The symmetry of the residuals plot provides validation of our early types being a (biased high) subset of the 9000 early types of Bernardi et al. (2003a).

The next step consists in substituting equations (7) into (4). To this extent, we have developed a mathematica code which combines equation (4) with equation (8). We obtain

$M_{\mathrm{BH}}=\alpha_{L} L+g_{0} \varepsilon_{L}$ 

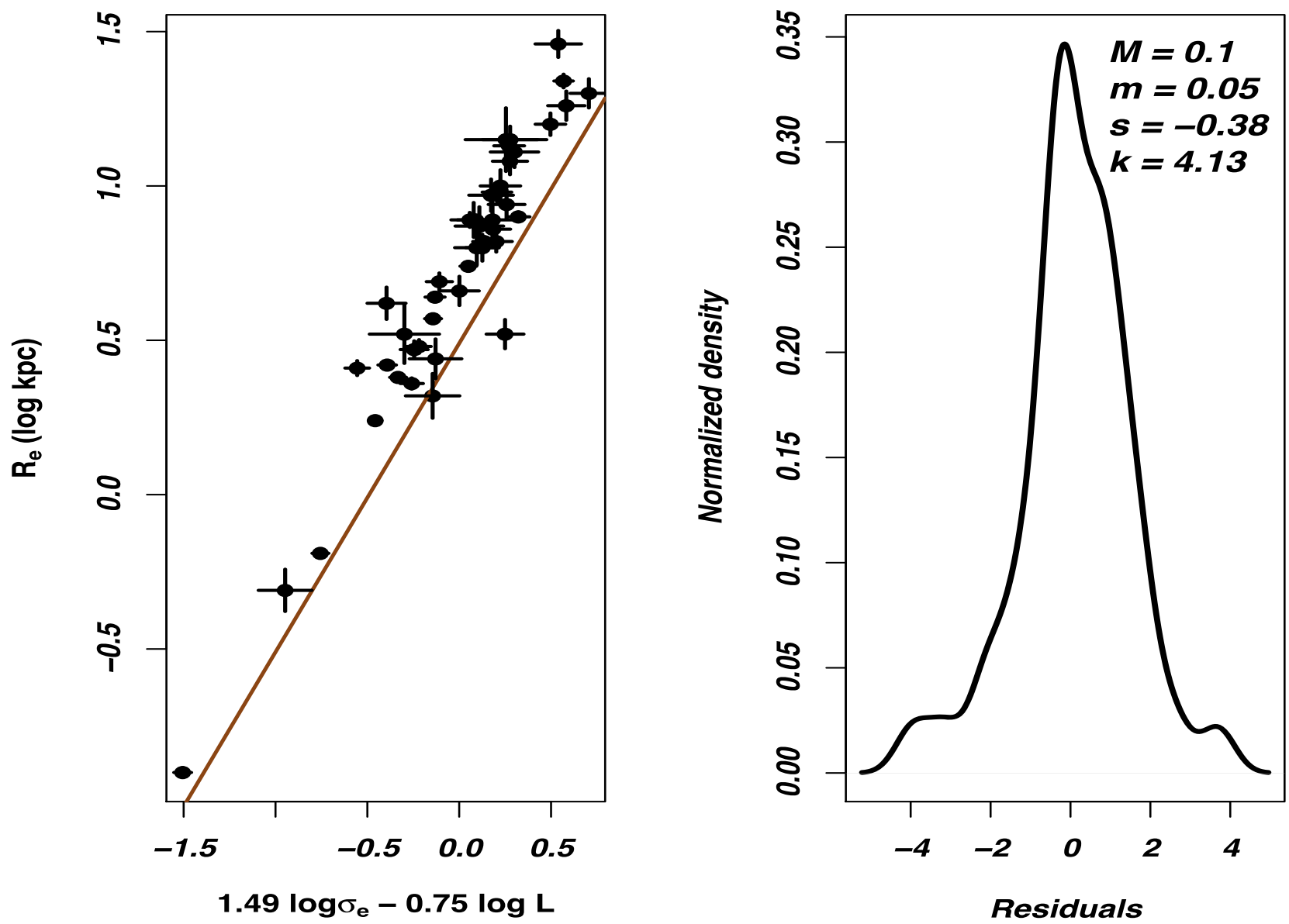

Figure 5. Left: Effective radius $R_{e}$ as a function of the FP relation found by Bernardi et al. (2003c). Our galaxies follow that FP, whose equation is reported on the $x$-axis, but are larger than expected. Right: Distribution of the residuals (normalized to unity) with respect to the new best-fitting line obtained by minimizing with respect to the normalization only. $M, m, s$, and $k$ are mean, median, skewness, and kurtosis, respectively. Both quantities on the axes are unitless.

where

$\alpha_{L}=A+\frac{B \sigma_{R_{e}} \rho_{R_{e} L}+C \sigma_{V} \rho_{V L}}{\sigma_{L}}$,

$\varepsilon_{L}=\sqrt{\Sigma^{2}+B^{2} \sigma_{R_{e}}^{2}\left(1-\rho_{R_{e} L}^{2}\right)+C^{2} \sigma_{V}^{2}\left(1-\rho_{V L}^{2}\right)+\Gamma_{L}}$,

$\Gamma_{L}=2 B C \sigma_{R_{e}} \sigma_{V}\left(\rho_{R_{e} V}-\rho_{L V} \rho_{R_{e} L}\right)$.

We have thus put together all the terms multiplying $L$ and all those multiplying the casual coefficients by adding them in quadrature, so that $\alpha_{L}$ is the slope of the relation and $\varepsilon_{L}$ its intrinsic scatter. In practice, we are deriving the analytic expression $\left(M_{\mathrm{BH}} \mid V, R_{e}\right)$ relation, i.e. we are projecting the hyperplanar relation of the previous paragraph on the $M_{\mathrm{BH}}-L$ relation. Because of the symmetry of the trivariate distribution, we can write analogous expressions to equation (11) by simply interchanging variables and coefficients. If we drew, say, $L$ and $V$ from $R_{e}$, then

$M_{\mathrm{BH}}=\alpha_{R_{e}} R_{e}+g_{0} \varepsilon_{R_{e}}$

where

$\alpha_{R_{e}}=B+\frac{A \sigma_{L} \rho_{R_{e} L}+C \sigma_{V} \rho_{V R_{e}}}{\sigma_{R_{e}}}$,

$\varepsilon_{R_{e}}=\sqrt{\Sigma^{2}+A^{2} \sigma_{L}^{2}\left(1-\rho_{R_{e} L}^{2}\right)+C^{2} \sigma_{V}^{2}\left(1-\rho_{V R_{e}}^{2}\right)+\Gamma_{R_{e}}}$,
$\Gamma_{R_{e}}=2 A C \sigma_{L} \sigma_{V}\left(\rho_{L V}-\rho_{R_{e} V} \rho_{R_{e} L}\right)$,

while starting from $V$ we would obtain

$M_{\mathrm{BH}}=\alpha_{V} V+g_{0} \varepsilon_{V}$

where

$\alpha_{V}=C+\frac{A \sigma_{L} \rho_{V L}+B \sigma_{R} \rho_{V R_{e}}}{\sigma_{V}}$,

$\varepsilon_{V}=\sqrt{\Sigma^{2}+A^{2} \sigma_{L}^{2}\left(1-\rho_{R_{e} V}^{2}\right)+B^{2} \sigma_{R_{e}}^{2}\left(1-\rho_{V R_{e}}^{2}\right)+\Gamma_{V}}$,

$\Gamma_{V}=2 A B \sigma_{L} \sigma_{R_{e}}\left(\rho_{R_{e} L}-\rho_{R_{e} V} \rho_{V L}\right)$.

Equations (12), (14), and (16) are those we are going to use to find the slopes of and the intrinsic scatters starting from the intrinsic relation (4) and taking the FP into account.

\subsection{The fundamental BH-hosts relation}

Using equations (12a), (14a), and (16a), we can compute the expected slopes for the three monovariate relations using the values of $A, B$, and $C$ from the hyperplanar regression, while equations (12b), (14b), and (16b) give us the three intrinsic scatters. However, considering the approximations we made (see par. 4.2), we preferred 
Table 5. Regression results between $M_{\mathrm{BH}}$ and quantities obtained from linear combinations of $\sigma_{e}$ and $R_{e}$. Column 1: the independent variable. Column 2: the subgroup. Column 3: the slope. Column 4: the zero-point. Column 5: the intrinsic scatter. Column 6: The value of $\langle x\rangle$ (equation 2). The subgroup $E T$ embeds galaxies flagged with $A=0$ (core-galaxies) or $A=1$ (power-law ellipticals, Table 1).

\begin{tabular}{lccccc}
\hline Variable(s) & Subgroup & $\alpha$ & $\beta$ & $\varepsilon$ & $\langle x\rangle$ \\
\hline$M_{\text {Hop }}$ & All & $0.99 \pm 0.05$ & $8.43 \pm 0.04$ & $0.37 \pm 0.04$ & 9.332 \\
& ClBul & $0.88 \pm 0.05$ & $8.85 \pm 0.04$ & $0.35 \pm 0.04$ & 9.504 \\
& $E T$ & $0.92 \pm 0.06$ & $8.85 \pm 0.04$ & $0.38 \pm 0.04$ & 9.689 \\
$M_{\text {vir }}$ & All & $0.88 \pm 0.05$ & $8.44 \pm 0.05$ & $0.46 \pm 0.05$ & 4.813 \\
& ClBul & $0.80 \pm 0.05$ & $8.83 \pm 0.04$ & $0.45 \pm 0.05$ & 4.903 \\
& $E T$ & $0.93 \pm 0.06$ & $8.84 \pm 0.04$ & $0.44 \pm 0.04$ & 5.012 \\
$U_{\text {grav }}$ & All & $0.69 \pm 0.04$ & $8.43 \pm 0.05$ & $0.41 \pm 0.04$ & 9.513 \\
& $C l B u l$ & $0.63 \pm 0.05$ & $8.83 \pm 0.04$ & $0.39 \pm 0.04$ & 9.679 \\
& $E T$ & $0.69 \pm 0.04$ & $8.84 \pm 0.04$ & $0.40 \pm 0.04$ & 9.824 \\
\hline & & & & &
\end{tabular}

to be conservative trying to simplify this approach by assuming one relation to be fundamental and see how it performs in predicting the others.

For example, let us assume $M_{\mathrm{BH}}-V$ to be the fundamental relation. If $V$ is the sole variable of importance in deriving $M_{\mathrm{BH}}$, then we should have $A=B=0$ in equation (4) and $C$ and $\Sigma$ equal to slope and intrinsic scatter of the $M_{\mathrm{BH}}-V$ (Table 2). We must set $A=B=0$ not only in equation (4), but also in equations (14) and (12). Then, by using for $C$ and $\Sigma$ the values obtained through the linear regression we can check how $V$ predicts slopes and intrinsic scatters of $M_{\mathrm{BH}}-L$ (using equations 12) and $M_{\mathrm{BH}}-R_{e}$ (using equations 14).

This procedure must then be repeated assuming, in turn, the other two relations to be fundamental. Besides these three photometric quantities appearing in our sample, the results of above suggest considering linear combinations of $V$ and $R_{e}$ too, i.e.

$W=a V+b R_{e}$

where $a$ and $b$ are integers which give $W$ a particular meaning. The three combinations examined here are as follows:

(i) $a=2$ and $b=1$, which make $W$ the mass predicted by the virial theorem $\left(M_{\mathrm{vir}}\right)$;

(ii) $a=4$ and $b=0.4\left(M_{\mathrm{Hop}}\right)$, which is the relation proposed by Hopkins et al. (2007b) as the fundamental relation with the coefficients derived in Table 2;

(iii) $a=4$ and $b=1$, which makes $W$ the gravitational energy $U_{\text {grav }}$ of a singular isothermal sphere (SIS).

These three quantities can be used in equation (4) just as $L$, $V$, and $R_{e}$. In particular, $M_{\mathrm{vir}}$ is expected to be a good proxy of $M_{\text {bul }}$ (Cappellari et al. 2006). The results of the regressions (using the whole sample) linking $M_{\mathrm{BH}}$ to these three new variables [equation (2)] are reported in Table 5. Apart from the $M_{\mathrm{BH}^{-}}$ $M_{\mathrm{Hop}}$, no relation is better than the $M_{\mathrm{BH}}-\sigma_{e}$ in terms of intrinsic scatter.

In order to assess the goodness of the predictions, a $\chi^{2}$ defined as

$\chi^{2}=\sum_{j}\left[\left(\frac{\alpha_{\mathrm{obs}}-\alpha_{\mathrm{mod}}}{\sigma_{\alpha, \mathrm{obs}}}\right)^{2}+\left(\frac{\varepsilon_{\mathrm{obs}}-\varepsilon_{\mathrm{mod}}}{\sigma_{\varepsilon, \mathrm{obs}}}\right)^{2}\right]$

has been used to compare the results predicted by a relation with those obtained from the fits. The subscripts obs and mod refer to the parameters derived from the regression and those computed with
Table 6. Comparison of the results obtained from the regressions and those obtained with the model described above only including early types with the covariance matrix of Bernardi et al. (2003b). Each row represents the model prediction assuming as fundamental variable that in the leftmost column. $\alpha$ 's and $\varepsilon$ 's are slopes and intrinsic scatters, respectively. The rightmost column shows the $\chi^{2}$ values. The values in bold are those obtained through linear regressions.

\begin{tabular}{lcccccccc}
\hline Fund. / Obs. & & $L$ & $R_{e}$ & $V$ & $M_{\mathrm{Hop}}$ & $M_{\mathrm{Vir}}$ & $U_{\mathrm{Grav}}$ & $\chi^{2}$ \\
\hline$L$ & $\alpha$ & $\mathbf{1 . 1 3}$ & 1.38 & 2.66 & 0.68 & 0.90 & 0.59 & 34.1 \\
& $\varepsilon$ & $\mathbf{0 . 4 4}$ & 0.47 & 0.50 & 0.47 & 0.45 & 0.46 & \\
$R_{e}$ & $\alpha$ & 0.73 & $\mathbf{1 . 1 4}$ & 1.35 & 0.43 & 0.64 & 0.40 & 150.5 \\
& $\varepsilon$ & 0.61 & $\mathbf{0 . 5 9}$ & 0.64 & 0.62 & 0.60 & 0.61 & \\
$V$ & $\alpha$ & 1.11 & 1.08 & $\mathbf{4 . 3 2}$ & 0.93 & 1.01 & 0.73 & 1.4 \\
& $\varepsilon$ & 0.50 & 0.57 & $\mathbf{0 . 4 1}$ & 0.41 & 0.47 & 0.44 & \\
$M_{\text {Hop }}$ & $\alpha$ & 1.24 & 1.48 & 4.07 & $\mathbf{0 . 9 2}$ & 1.07 & 0.74 & 7.4 \\
& $\varepsilon$ & 0.43 & 0.48 & 0.39 & $\mathbf{0 . 3 8}$ & 0.41 & 0.39 & \\
$M_{\text {Vir }}$ & $\alpha$ & 1.10 & 1.49 & 2.96 & 0.71 & $\mathbf{0 . 9 3}$ & 0.62 & 25.1 \\
& $\varepsilon$ & 0.45 & 0.46 & 0.48 & 0.46 & $\mathbf{0 . 4 4}$ & 0.45 & \\
$U_{\text {Grav }}$ & $\alpha$ & 1.20 & 1.53 & 3.53 & 0.82 & 1.02 & $\mathbf{0 . 6 9}$ & 11.2 \\
& $\varepsilon$ & 0.42 & 0.45 & 0.42 & 0.40 & 0.40 & $\mathbf{0 . 4 0}$ & \\
\hline & & & & & & & &
\end{tabular}

our model, respectively. The variances of the three new variables of Table 5 are linked to those of $V$ and $R_{e}$ by the notorious formula

$\sigma_{W}^{2}=a^{2} \sigma_{V}^{2}+b^{2} \sigma_{R_{e}}^{2}+2 a b \sigma_{V} \sigma_{R} \rho_{V R_{e}}$.

The results for the sample of early-type galaxies are shown Table 6. $V$ predicts the other relations better than its linear combinations with $R_{e}$ or the other two monovariate correlations. It is intriguing that, even though $R_{e}$ alone completely fails in predicting the coefficients of the other regressions, increasing its exponent from $0.4\left(M_{\mathrm{Hop}}\right)$ to $1\left(U_{\text {grav }}\right)$ does not change the $\chi^{2}$ significantly, showing that $V$ is the predominant variable.

We now repeat the analysis considering all classical bulges, i.e. also those coming from decompositions of spirals. Although we do not have a covariance matrix that describes such a sample, we can still take the covariance matrix of Bernardi et al. (2003b), since classical bulges behave in the same way as early types. We caution that in this case all the errors coming from the decompositions are additional sources of uncertainty for the results that follow, even though we will show in the next paragraph that what really makes the difference for the results is the covariance matrix.

Taking again the values of Table 4 to build the covariance matrix, we obtain the results reported in Table 7 . We see that the only difference with respect to the previous paragraph is that here the results of $M_{\mathrm{Hop}}$ and $U_{\text {grav }}$ are interchanged. But even in this case it is the velocity dispersion to yield the best predictions of the other relations.

\subsection{The importance of the covariance matrix}

We now show the critical dependence on the covariance matrix of the results we obtained in the last section. We have in fact used a covariance matrix from the SDSS sample of early-type galaxies that is more homogeneous and larger than ours which is also biased towards more luminous objects (Shankar et al. 2016, Fig. 5). Thus, we show the consequences of using, at least, the variances obtained 
Table 7. Same as Table 6 also including bulges coming from decompositions of spirals.

\begin{tabular}{lcccccccc}
\hline Fund. / Obs. & & $L$ & $R_{e}$ & $V$ & $M_{\text {Hop }}$ & $M_{\text {Vir }}$ & $U_{\text {Grav }}$ & $\chi^{2}$ \\
\hline$L$ & $\alpha$ & $\mathbf{1 . 0 0}$ & 1.18 & 2.27 & 0.57 & 0.77 & 0.50 & 86.4 \\
& $\varepsilon$ & $\mathbf{0 . 4 7}$ & 0.50 & 0.51 & 0.50 & 0.48 & 0.48 & \\
$R_{e}$ & $\alpha$ & 0.57 & $\mathbf{0 . 9 1}$ & 1.05 & 0.33 & 0.50 & 0.31 & 293.7 \\
& $\varepsilon$ & 0.62 & $\mathbf{0 . 6 0}$ & 0.64 & 0.63 & 0.62 & 0.62 & \\
$V$ & $\alpha$ & 1.12 & 1.08 & $\mathbf{4 . 4 8}$ & 0.93 & 0.96 & 0.71 & 14.6 \\
& $\varepsilon$ & 0.48 & 0.55 & $\mathbf{0 . 3 8}$ & 0.40 & 0.47 & 0.43 & \\
$M_{\text {Hop }}$ & $\alpha$ & 1.18 & 1.40 & 3.89 & $\mathbf{0 . 8 8}$ & 1.01 & 0.71 & 37.2 \\
& $\varepsilon$ & 0.40 & 0.45 & 0.36 & $\mathbf{0 . 3 5}$ & 0.38 & 0.36 & \\
$M_{\text {Vir }}$ & $\alpha$ & 0.95 & 1.28 & 2.39 & 0.60 & $\mathbf{0 . 8 0}$ & 0.53 & 76.7 \\
& $\varepsilon$ & 0.46 & 0.47 & 0.49 & 0.47 & $\mathbf{0 . 4 5}$ & 0.46 & \\
$U_{\text {Grav }}$ & $\alpha$ & 1.10 & 1.41 & 3.15 & 0.75 & 0.94 & $\mathbf{0 . 6 3}$ & 42.5 \\
& $\varepsilon$ & 0.40 & 0.43 & 0.42 & 0.39 & 0.39 & $\mathbf{0 . 3 9}$ & \\
\hline
\end{tabular}

Table 8. Variances obtained from our classic bulge sample by fitting equation (10) to it and taking correlation coefficients from Table 4.

\begin{tabular}{lc}
\hline Coefficient & Value \\
\hline$\sigma_{V}$ & $0.17 \pm 0.01$ \\
$\sigma_{L}$ & $0.64 \pm 0.03$ \\
$\sigma_{R_{e}}$ & $0.55 \pm 0.03$ \\
\hline
\end{tabular}

Table 9. Same as Table 7 using variances directly derived from our sample.

\begin{tabular}{lcccccccc}
\hline Fund. / Obs. & & $L$ & $R_{e}$ & $V$ & $M_{\text {Hop }}$ & $M_{\text {Vir }}$ & $U_{\text {Grav }}$ & $\chi^{2}$ \\
\hline$L$ & $\alpha$ & $\mathbf{1 . 0 0}$ & 1.03 & 2.93 & 0.69 & 0.79 & 0.56 & 39.9 \\
& $\varepsilon$ & $\mathbf{0 . 4 7}$ & 0.55 & 0.61 & 0.55 & 0.49 & 0.51 & \\
$R_{e}$ & $\alpha$ & 0.67 & $\mathbf{0 . 9 1}$ & 1.55 & 0.45 & 0.58 & 0.39 & 203.3 \\
& $\varepsilon$ & 0.65 & $\mathbf{0 . 6 0}$ & 0.73 & 0.68 & 0.62 & 0.65 & \\
$V$ & $\alpha$ & 0.89 & 0.73 & $\mathbf{4 . 4 8}$ & 0.87 & 0.76 & 0.61 & 3.3 \\
& $\varepsilon$ & 0.59 & 0.72 & $\mathbf{0 . 3 8}$ & 0.42 & 0.57 & 0.49 & \\
$M_{\text {Hop }}$ & $\alpha$ & 1.00 & 1.02 & 4.18 & $\mathbf{0 . 8 9}$ & 0.86 & 0.66 & 2.5 \\
& $\varepsilon$ & 0.48 & 0.59 & 0.39 & $\mathbf{0 . 3 5}$ & 0.44 & 0.38 & \\
$M_{\text {Vir }}$ & $\alpha$ & 0.95 & 1.09 & 3.03 & 0.71 & $\mathbf{0 . 8 0}$ & 0.58 & 33.9 \\
& $\varepsilon$ & 0.48 & 0.50 & 0.58 & 0.51 & $\mathbf{0 . 4 5}$ & 0.47 & \\
$U_{\text {Grav }}$ & $\alpha$ & 1.02 & 1.11 & 3.66 & 0.82 & 0.86 & $\mathbf{0 . 6 3}$ & 10.7 \\
& $\varepsilon$ & 0.44 & 0.51 & 0.49 & 0.41 & 0.40 & $\mathbf{0 . 3 9}$ & \\
\hline
\end{tabular}

from our sample through a maximum likelihood analysis such as the one presented in the last section. ${ }^{7}$

We start by considering all classical bulges of our sample. Taking the correlation coefficients from Table 4 and fitting the three variances using equation (10), we get the covariance matrix reported in Table 8, which leads to the results for our analysis of Table 9.

This shows how critical the choice of the covariance matrix turns out to be. In fact, using the variances from our sample, the BHFP has a $\chi^{2}$ slightly lower than that of $V$. When instead we just use the early types of our sample, the covariance matrix (Table 10) leads to the results reported in Table 11.

\footnotetext{
${ }^{7}$ Note that, given the small number of data points, the estimate of the whole covariance matrix from our sample could lead to huge errors.
}

Table 10. Same as Table 8 only including early types.

\begin{tabular}{lc}
\hline Coefficient & Value \\
\hline$\sigma_{V}$ & $0.16 \pm 0.01$ \\
$\sigma_{L}$ & $0.58 \pm 0.04$ \\
$\sigma_{R_{e}}$ & $0.46 \pm 0.03$ \\
\hline
\end{tabular}

Table 11. Same as Table 9 using early types only.

\begin{tabular}{lcccccccc}
\hline Fund. / Obs. & & $L$ & $R_{e}$ & $V$ & $M_{\mathrm{Hop}}$ & $M_{\text {Vir }}$ & $U_{\text {Grav }}$ & $\chi^{2}$ \\
\hline$L$ & $\alpha$ & $\mathbf{1 . 1 3}$ & 1.25 & 3.21 & 0.79 & 0.92 & 0.64 & 11.6 \\
& $\varepsilon$ & $\mathbf{0 . 4 4}$ & 0.54 & 0.60 & 0.52 & 0.47 & 0.48 & \\
$R_{e}$ & $\alpha$ & 0.81 & $\mathbf{1 . 1 4}$ & 1.80 & 0.54 & 0.72 & 0.48 & 88.5 \\
& $\varepsilon$ & 0.64 & $\mathbf{0 . 5 9}$ & 0.74 & 0.68 & 0.62 & 0.65 & \\
$V$ & $\alpha$ & 0.93 & 0.81 & $\mathbf{4 . 3 2}$ & 0.90 & 0.86 & 0.66 & 7.7 \\
& $\varepsilon$ & 0.59 & 0.71 & $\mathbf{0 . 4 1}$ & 0.43 & 0.54 & 0.48 & \\
$M_{\text {Hop }}$ & $\alpha$ & 1.09 & 1.17 & 4.30 & $\mathbf{0 . 9 2}$ & 0.95 & 0.71 & 0.2 \\
& $\varepsilon$ & 0.48 & 0.58 & 0.40 & $\mathbf{0 . 3 8}$ & 0.45 & 0.40 & \\
$M_{\text {Vir }}$ & $\alpha$ & 1.07 & 1.30 & 3.48 & 0.80 & $\mathbf{0 . 9 3}$ & 0.65 & 8.4 \\
& $\varepsilon$ & 0.47 & 0.49 & 0.55 & 0.49 & $\mathbf{0 . 4 4}$ & 0.45 & \\
$U_{\text {Grav }}$ & $\alpha$ & 1.11 & 1.27 & 3.94 & 0.88 & 0.96 & $\mathbf{0 . 6 9}$ & 2.0 \\
& $\varepsilon$ & 0.45 & 0.51 & 0.47 & 0.42 & 0.41 & $\mathbf{0 . 4 0}$ & \\
\hline
\end{tabular}

In this last case, both the BHFP and $U_{\text {grav }}$ reproduce almost perfectly the other relations, while $V$ has a higher $\chi^{2}$ than all its linear combination with $R_{e}$. This can be explained by the fact that the Kormendy relation we derived for our sample has a much lower intrinsic scatter than in other works on this topic (e.g. Saglia et al. 2016). The quantity $V$ alone yields the worst predictions for the monovariate correlations $M_{\mathrm{BH}}-L$ and $M_{\mathrm{BH}}-R_{e}$, while this estimate improves drastically when $V$ gets combined with $R_{e}$. Note that despite the reduced number of data-points (49 for the early-type subsample) the errors in the variance estimates reassure us about the robustness of the minimization results. Thus, we caution that using a covariance matrix estimated from a biased and heterogeneous sample can significantly alter the results of the analysis.

\section{CONCLUSIONS}

We have studied the scaling relations between SMBHs and their host galaxies, extending our analysis to the four-dimensional case. In this work, we analytically combine for the first time the whole FP with $\mathrm{BH}$ masses deriving formulae to express slopes and intrinsic scatter of the $\mathrm{BH}-$ hosts as functions of the covariance matrix. Conversely to the findings of Hopkins et al. (2007b), the fundamental scaling relation seems to be the canonical $M_{\mathrm{BH}}-\sigma_{e}$, even though a bivariate relation $M_{\mathrm{BH}} \propto \sigma_{e}^{\sim 4} R_{e}^{\sim \beta}$ with an exponent $0.4 \leq \beta \leq 1$ acceptably explains the other correlations when combined with the FP. We have seen that this result is independent of whether we include or not classical bulge parameters coming from decompositions (but the same also holds for pseudo-bulges, see Appendix B) but also that it critically depends on the covariance matrix one chooses out for the analysis.

In the only other work where the whole FP is taken into account (van den Bosch 2016) the main conclusion also points to a $\mathrm{BH}-$ host coevolution driven by $\sigma_{e}$ solely. Indeed, the intrinsic scatter of the $M_{\mathrm{BH}}-\sigma_{e}$ relation is not significantly improved by higher dimensionality (Section 3). In the work of van den Bosch (2016), no improvement at all is found, but in that work the focus is on 
whole galaxies rather than decomposition, and $\mathrm{BHs}$ are known to correlate poorly (if at all) with disc parameters (Kormendy et al. 2011).

Provided that each galaxies hosts, or has hosted, an AGN at its centre (Soltan 1982), we believe that the $M_{\mathrm{BH}}-\sigma_{e}$ relation is established when $M_{\mathrm{BH}}$ reaches a critical value $M_{\sigma}$ proportional to $\sigma_{e}^{4}$ which signals a change of the AGN feedback from momentumdriven to energy-driven (King 2003, 2005). While in the first case the efficient Compton cooling enables SMBHs and bulges to coevolve pacifically, in the second case the energy output from $\mathrm{BH}$ winds is two orders of magnitude larger than the bulge's binding energy, thus seriously threatening the integrity of the host spheroid (King \& Pounds 2015). However, the simple $M_{\sigma} \propto \sigma_{e}^{4}$ dependence arises from the (unrealistic) description of a galaxy as a singular isothermal sphere (SOI, see equations 37-41 of King \& Pounds 2015). If the potential has a more complicated form, then a dependence on $R_{e}$ might come out, but the whole picture is still uncertain. Furthermore, since the modelling of the FP as a trivariate Gaussian (Bernardi et al. 2003b) introduces covariances and correlations between the bulge parameters and considering how tight this relation is, then it can be expected that a bivariate correlation can provide acceptable results. Besides, as we have seen in Section 4.4, the results differ depending on the variances, so the whole picture is still uncertain.

These problems could be resolved by the future development of new generation facilities. For instance, the sample used in this work is very heterogeneous regardless of the variable we consider. This is of particular relevance for $\mathrm{BH}$ mass estimates, which challenge current-day facilities and often give disagreeing results when different techniques are applied to the same galaxy (Kormendy \& Ho 2013), and, furthermore, the subset of BH masses nowadays available are likely to be a biased-high subsample (Shankar et al. 2016, 2017, 2019). Velocity dispersions should also be measured with the same instrument, but such coverage is not available. As far as concerns photometry, decomposing all spirals of our sample ( $\sim 60)$ using $K$-band data (Spitzer data are not available for the whole sample) steps beyond the purposes of this work, and more accurate multicomponent decompositions could lead to significantly different results (see e.g. the latest results of Davis, Graham \& Cameron 2018, 2019; Sahu, Graham \& Davis 2019).

We finally note that the fact that we assume each relation to be the fundamental one in order to see how it performs in predicting the others is itself an approximation. We should use the whole equations (12), (14), and (16) to quantify the correction introduced by the FP on each of the monovariate scaling relations.

\section{ACKNOWLEDGEMENTS}

We thank the anonymous referee for comments on the manuscript. GL acknowledges support from the European Union's Horizon 2020 Sundial Innovative Training Network, grant no. 721463. This work has made use of the HyperLeda data base (Paturel et al. 2003).

\section{REFERENCES}

Beifiori A., Courteau S., Corsini E. M., Zhu Y., 2012, MNRAS, 419, 2497 Bernardi M. et al., 2003a, AJ, 125, 1817

Bernardi M. et al., 2003b, AJ, 125, 1849

Bernardi M. et al., 2003c, AJ, 125, 1866

Bernardi M., Sheth R. K., Tundo E., Hyde J. B., 2007, ApJ, 660, 267
Cappellari M. et al., 2006, MNRAS, 366, 1126

Cappellari M. et al., 2008, in Bureau M., Athanassoula E., Barbuy B., eds, Proc. IAU Symp. 245, Formation and Evolution of Galaxy Bulges, p. 215

Cappellari M. et al., 2013, MNRAS, 432, 1709

Davis B. L., Graham A. W., Seigar M. S., 2017, MNRAS, 471, 2187

Davis B. L., Graham A. W., Cameron E., 2018, ApJ, 869, 113

Davis B. L., Graham A. W., Cameron E., 2019, ApJ, 873, 85

Djorgovski S., Davis M., 1987, ApJ, 313, 59

Erwin P. et al., 2015, MNRAS, 446, 4039

Feoli A., Mancini L., 2009, ApJ, 703, 1502

Ferrarese L., 2002, ApJ, 578, 90

Ferrarese L., Merritt D., 2000, ApJ, 539, L9

Fisher D. B., Drory N., 2008, AJ, 136, 773

Fisher D. B., Bolatto A., Drory N., Combes F., Blitz L., Wong T., 2013, ApJ, 764,174

Gebhardt K. et al., 2000, ApJ, 539, L13

Graham A. W., 2014, in Seigar M. S., Treuthardt P., eds, ASP Conf. Ser. Vol. 480, Structure and Dynamics of Disk Galaxies. Astron. Soc. Pac., San Francisco, p. 185

Graham A. W., 2016, in Laurikainen E., Peletier R., Gadotti D., eds, Astrophysics and Space Science Library, Vol. 418, Galactic Bulges. Springer-Verlag, Berlin,p. 263

Graham A. W., Scott N., 2013, ApJ, 764, 151

Gültekin K. et al., 2009, ApJ, 698, 198

Häring N., Rix H.-W., 2004, ApJ, 604, L89

Hopkins P. F., Hernquist L., Cox T. J., Robertson B., Krause E., 2007a, ApJ, 669,45

Hopkins P. F., Hernquist L., Cox T. J., Robertson B., Krause E., 2007b, ApJ, 669,67

Kelly B. C., 2007, ApJ, 665, 1489

King A., 2003, ApJ, 596, L27

King A., 2005, ApJ, 635, L121

King A., Pounds K., 2015, ARA\&A, 53, 115

Kondratko P. T., Greenhill L. J., Moran J. M., 2005, ApJ, 618, 618

Kormendy J., Bender R., 2011, Nature, 469, 377

Kormendy J., Ho L. C., 2013, ARA\&A, 51, 511

Kormendy J., Kennicutt R. C., Jr, 2004, ARA\&A, 42, 603

Kormendy J., Bender R., Cornell M. E., 2011, Nature, 469, 374

Krajnović D. et al., 2018, MNRAS, 477, 3030

Kuo C. Y. et al., 2011, ApJ, 727, 20

Magorrian J. et al., 1998, AJ, 115, 2285

Mancini L., Feoli A., 2012, A\&A, 537, A48

Marconi A., Hunt L. K., 2003, ApJ, 589, L21

McConnell N. J., Ma C.-P., 2013, ApJ, 764, 184

Paturel G., Petit C., Prugniel P., Theureau G., Rousseau J., Brouty M., Dubois P., Cambrésy L., 2003, A\&A, 412, 45

Rousseeuw P. J., Van Driessen K., 2006, Data Min. Knowl. Discovery, 12, 29

Rusli S. P. et al., 2013, AJ, 146, 45

Saglia R. P., Colless M., Burstein D., Davies R. L., McMahan R. K., Wegner G., 2001, MNRAS, 324, 389

Saglia R. P. et al., 2016, ApJ, 818, 47

Sahu N., Graham A. W., Davis B. L., 2019, ApJ, 876, 155

Sani E., Marconi A., Hunt L. K., Risaliti G., 2011, MNRAS, 413, 1479

Savorgnan G. A. D., Graham A. W., 2016, ApJS, 222, 10

Savorgnan G., Graham A. W., Marconi A., Sani E., Hunt L. K., Vika M., Driver S. P., 2013, MNRAS, 434, 387

Savorgnan G. A. D., Graham A. W., Marconi A., Sani E., 2016, ApJ, 817, 21

Schulze A., Gebhardt K., 2011, ApJ, 729, 21

Shankar F. et al., 2016, MNRAS, 460, 3119

Shankar F., Bernardi M., Sheth R. K., 2017, MNRAS, 466, 4029

Shankar F. et al., 2019, MNRAS, 485, 1278

Soltan A., 1982, MNRAS, 200, 115

Tremaine S. et al., 2002, ApJ, 574, 740

van den Bosch R. C. E., 2016, ApJ, 831, 134

Yamauchi A., Nakai N., Sato N., Diamond P., 2004, PASJ, 56, 605 


\section{APPENDIX A: NOTES ON DISCARDED GALAXIES}

Galaxies omitted because of incomplete data. The following objects were omitted because of the unavailability of photometric measurements in the infrared: Milky Way, ${ }^{8}$ NGC1194, NGC4526, NGC6264, NGC6323.

NGC3607: We follow Kormendy \& Ho (2013) in omitting this galaxy because, in this case, not allowing for a DM halo yields underestimated results because the BH SOI is not well resolved.

NGC4388: The $M_{\mathrm{BH}}$ value is uncertain because of the lack of a systemic maser in this galaxy and because the rotation might even be non-Keplerian (Kuo et al. 2011). The velocity dispersion is uncertain since it neglects rotation inside the effective radius of the pseudo-bulge [see Kormendy \& Ho (2013)]. Moreover, Savorgnan \& Graham (2016) report complications due to dust absorption when deriving the photometry.

NGC2974, NGC3414, NGC4552 (M89), NGC4621 (M59), NGC5813, NGC5846: These (uncertain) $M_{\mathrm{BH}} \mathrm{s}$ come from an unlabelled plot from Cappellari et al. (2008), who do not provide any information or details about the observations and data analysis. We thus consider the $\mathrm{BH}$ masses unreliable.

NGC3079: We reject the $\mathrm{BH}$ mass value because the rotation curve is flat (Kondratko, Greenhill \& Moran 2005). Saglia et al. (2016) accept the galaxy stating that the estimate agrees with Yamauchi et al. (2004). However, both authors do not provide an exact $M_{\mathrm{BH}}$ value. This galaxy also shows a steep drop in $\sigma_{e}$ in the central regions, probably because of bar streaming motions (Graham \& Scott 2013)

NGC4486B: We follow Saglia et al. (2016), who omit the galaxy because of the abnormally large $\mathrm{BH}$ mass. Indeed, this galaxy is well known to be an outlier (Gültekin et al. 2009). Moreover, the $M_{\mathrm{BH}}$ value was derived without allowing for a DM halo and comes from unpublished literature (Saglia et al. 2016).

$N G C 4736, N G C 4826$ : We reject these (commonly accepted) $M_{\mathrm{BH}}$ estimates because details on the observation are not provided (see Kormendy et al. 2011).

NGC1300: This galaxy appears to have an uncertain velocity dispersion. In fact, the value from Saglia et al. (2016) is much lower than that reported in the sample of van den Bosch (2016). Using the first value would make this galaxy the largest outlier in the $M_{\mathrm{BH}}-\sigma_{e}$ relation, while, interestingly, van den Bosch (2016) reports the second value to be too high.

NGC2787: This galaxy hosts both a classical and a pseudo-bulge. Since it is unclear what Savorgnan \& Graham (2016) model in their analysis, even if the pseudo-bulge seems to be more prominent (Kormendy \& Ho 2013), we do not trust the photometry and omit this galaxy.

NGC2960: We omit this galaxy because of its extremely uncertain morphology. In fact, Saglia et al. (2016) classify the galaxy as an E2 but give it a $T$ flag value of 3 , which would make this galaxy a pure pseudo-bulge.

IC1481: We follow Saglia et al. (2016) in omitting this galaxy because of a merger in progress which prevents the determination of a reliable photometric profile.

${ }^{8}$ For our own Galaxy, a $K$-band magnitude value can be found in Kormendy \& Ho (2013), whom, however, do not provide effective radii.

\section{APPENDIX B : RESULTS INCLUDING PSEUDO-BULGES}

This appendix contains the results of the analysis described in Section 4 including pseudo-bulges, which do not seem to correlate with their SMBHs (cf. Section 3.1). The covariance matrix for this case is reported in Table B1. By computing the analogous of Table 6 (Table B2) and of Table 11 (Table B3) we see that, although in this case the usage of the covariance matrix is not so well justified as pseudo-bulges form stars and thus have a much broader range of colours than classical bulges, no significant differences are found.

Table B1. Same as Table 8 using the full sample.

\begin{tabular}{lc}
\hline Coefficient & Value \\
\hline$\sigma_{V}$ & $0.18 \pm 0.01$ \\
$\sigma_{L}$ & $0.69 \pm 0.03$ \\
$\sigma_{R_{e}}$ & $0.64 \pm 0.04$ \\
\hline
\end{tabular}

Table B2. Same as Table B3 using the covariance matrix reported in Table 4.

\begin{tabular}{lcccccccc}
\hline Fund. / Obs. & & $L$ & $R_{e}$ & $V$ & $M_{\text {Hop }}$ & $M_{\text {Vir }}$ & $U_{\text {Grav }}$ & $\chi^{2}$ \\
\hline$L$ & $\alpha$ & $\mathbf{1 . 1 2}$ & 1.39 & 2.67 & 0.68 & 0.89 & 0.59 & 118.1 \\
& $\varepsilon$ & $\mathbf{0 . 5 1}$ & 0.54 & 0.56 & 0.54 & 0.52 & 0.53 & \\
$R_{e}$ & $\alpha$ & 0.69 & $\mathbf{1 . 0 7}$ & 1.28 & 0.43 & 0.62 & 0.39 & 413.4 \\
& $\varepsilon$ & 0.63 & $\mathbf{0 . 6 3}$ & 0.66 & 0.64 & 0.62 & 0.63 & \\
$V$ & $\alpha$ & 1.26 & 1.22 & $\mathbf{5 . 0 7}$ & 1.05 & 1.14 & 0.82 & 41.8 \\
& $\varepsilon$ & 0.53 & 0.63 & $\mathbf{0 . 4 2}$ & 0.43 & 0.49 & 0.46 & \\
$M_{\text {Hop }}$ & $\alpha$ & 1.31 & 1.68 & 4.28 & $\mathbf{0 . 9 9}$ & 1.13 & 0.79 & 70.6 \\
& $\varepsilon$ & 0.42 & 0.45 & 0.38 & $\mathbf{0 . 3 7}$ & 0.39 & 0.37 & \\
$M_{\text {Vir }}$ & $\alpha$ & 1.03 & 1.43 & 2.78 & 0.68 & $\mathbf{0 . 8 8}$ & 0.58 & 117.4 \\
& $\varepsilon$ & 0.47 & 0.46 & 0.49 & 0.47 & $\mathbf{0 . 4 6}$ & 0.46 & \\
$U_{\text {Grav }}$ & $\alpha$ & 1.18 & 1.59 & 3.48 & 0.82 & 1.02 & $\mathbf{0 . 6 9}$ & 66.2 \\
& $\varepsilon$ & 0.42 & 0.43 & 0.43 & 0.40 & 0.40 & $\mathbf{0 . 4 1}$ & \\
\hline
\end{tabular}

Table B3. Same as Table 9 using the full sample and the variances of Table B1.

\begin{tabular}{lcccccccc}
\hline Fund. / Obs. & & $L$ & $R_{e}$ & $V$ & $M_{\mathrm{Hop}}$ & $M_{\mathrm{Vir}}$ & $U_{\mathrm{Grav}}$ & $\chi^{2}$ \\
\hline$L$ & $\alpha$ & $\mathbf{1 . 1 2}$ & 1.08 & 3.33 & 0.78 & 0.84 & 0.61 & 49.7 \\
& $\varepsilon$ & $\mathbf{0 . 5 3}$ & 0.63 & 0.71 & 0.61 & 0.56 & 0.57 & \\
$R_{e}$ & $\alpha$ & 0.90 & $\mathbf{1 . 0 7}$ & 2.08 & 0.65 & 0.76 & 0.53 & 189.4 \\
& $\varepsilon$ & 0.70 & $\mathbf{0 . 6 3}$ & 0.86 & 0.73 & 0.65 & 0.68 & \\
$V$ & $\alpha$ & 1.01 & 0.76 & $\mathbf{5 . 0 7}$ & 0.97 & 0.86 & 0.68 & 11.4 \\
& $\varepsilon$ & 0.69 & 0.86 & $\mathbf{0 . 4 2}$ & 0.45 & 0.61 & 0.53 & \\
$M_{\text {Hop }}$ & $\alpha$ & 1.14 & 1.13 & 4.64 & $\mathbf{0 . 9 9}$ & 0.93 & 0.71 & 1.7 \\
& $\varepsilon$ & 0.52 & 0.60 & 0.40 & $\mathbf{0 . 3 7}$ & 0.45 & 0.39 & \\
$M_{\text {Vir }}$ & $\alpha$ & 1.10 & 1.18 & 3.68 & 0.83 & $\mathbf{0 . 8 8}$ & 0.64 & 27.8 \\
& $\varepsilon$ & 0.51 & 0.50 & 0.61 & 0.52 & $\mathbf{0 . 4 6}$ & 0.47 & \\
$U_{\text {Grav }}$ & $\alpha$ & 1.14 & 1.19 & 4.18 & 0.91 & 0.92 & $\mathbf{0 . 6 9}$ & 8.9 \\
& $\varepsilon$ & 0.47 & 0.51 & 0.50 & 0.42 & 0.41 & $\mathbf{0 . 4 1}$ & \\
\hline
\end{tabular}

This paper has been typeset from a $\mathrm{T}_{\mathrm{E}} \mathrm{X} / \mathrm{LT}_{\mathrm{E}} \mathrm{X}$ file prepared by the author. 